\title{
Analysis of Action Oriented Effects on Perceptual Process of Object Recognition Using Physiological Responses
}

\author{
Shanu Sharma ${ }^{1}$, Anju Mishra ${ }^{1}$, Sanjay Kumar ${ }^{2}$, Priya Ranjan ${ }^{3}$, and Amit \\ Ujlayan $^{4}$ \\ ${ }^{1}$ Department of CSE, ASET, Amity University, Uttar Pradesh, India, \\ shanu.sharma16@gmail.com, \\ ${ }^{1}$ Department of IT, ASET, Amity University, Uttar Pradesh, India, \\ amishra1@amity.edu, \\ ${ }^{2}$ Department of Psychology, Oxford Brookes University,U.K, \\ skumar@brookes.ac.uk, \\ ${ }^{3}$ Department of EEE, ASET, Amity University, Uttar Pradesh, India, \\ pranjan@amity.edu, \\ ${ }^{4}$ School of Vocational Studies and Applied Sciences, GBU, Greater Noida, \\ amitujlayan@gbu.ac.in
}

\begin{abstract}
Action on any objects provides perceptual information about the environment. There is a significant evidence that human visual system responds to action possibilities in an image as perceiving any ones action stimulates human motor system. However very limited studies have been done to analyze the effect of object affordance during action perception and execution. To study the effect of object affordance on human perception, in this paper we have analyzed the human brain signals using EEG based oscillatory activity of brain. EEG responses corresponding to images of objects shown with correct, incorrect and without grips are examined. Exploration of different gripping effects has been done by extracting Alpha and Beta frequency bands using Discrete Wavelet Transform based band extraction method, then baseline normalized power of Alpha and Beta frequency bands at 24 positions of motor area of left and right side of brain are examined. The result shows that 12 pooled electrodes at central and central parietal region provides a clear discrimination among the three gripping cases in terms of calculated power. The presented research explores new applicabilities of object affordance to develop a variety of Brain Computer Interface (BCI) based devices and to improve motor imagery ability among motor disorder related patients.
\end{abstract}

Keywords: Visual Perception, Action Recognition, Congruent-InCongruent Grip, EEG Signals, DWT

\section{Introduction}

Action is a way of obtaining perceptual knowledge about the environment. According to Gibson 1979 Visual affordance theory [1], any movement of body 
either active or passive can provide useful information. Donald Norman (1988) [2] explained affordance in context of human computer interaction as how an object may be interacted with. It indicates that not only the objects properties but the way we interact with the objects also affects our motor system $[3,10]$. So, Perception is instrumentally dependent on action and movement can alter sensory inputs and so result in different perceptions [1]. There is substantial indication that execution and observation of action both activates the same brain area and results in the same cognitive process. During planning, controlling or execution of movement, the motor cortex region of cerebral cortex is usually active. Thus, any activity related to movement or imagined movement generates brain signals that are dominant in the motor cortex of the brain $[8,9]$. Movement related oscillating process in these regions is usually visible in Alpha $(8-12 \mathrm{~Hz})$ and Beta $(12-30 \mathrm{~Hz})$ frequency bands[12,13]. To study the brain activity now a days brain computer interfaces (BCIs) is the most effective way. It detects and interprets signals and can generate commands to control external devices [4]. There are numerous applications of BCIs including medical, industrial, experimental psychology and neuro-rehabilitation etc., which are working on analyzing the sensory inputs $[4,6]$. Brain signals can be detected by capturing the electrical activity of the brain. The most effective way to understand the functioning of brain is by EEG-based oscillatory activity $[7,8]$. The EEG recordings contain cortical potentials which occur during various mental processes [9]. The main issue with BCI devices is to capture the human mental activities by analyzing EEG based brain waves. The development of BCI based applications is challenging task as brain waves must respond to surrounding environment in real time [4].

It is widely known phenomenon that EEG rhythmic activities occurs over motor related areas of brain while performing or imaging any movement related work [12,21]. Different spatio-temporal pattern of EEG can be predicted depending on the type of imagery performed as imagining motor actions can moderate the motor related rhythm and result in power changes [22] [23]. Over the past years a lot of work has been done in the field of motor imagery based development of BCI devices. In [6] a general architecture for the motor imagery signal classification for BCI devices is presented. [14] have proposed an efficient EEG classification technique to address the issue of inter-subject variability. Similar work in the field of hand movement classification using motor imagery based EEG signals also exists $[16,18,19]$.

According to the perceptual studies it is observed that humans are more attentive towards target oriented stimuli [10]. Thus imaging an action oriented object recognition task can provide better performance in various BCI or rehabilitation related applications. Some studies exists which explored effect of object affordance on human perception. In [3] authors explained theoretical concept behind the role of way of interaction with object while observing the action, whereas analysis of the effects is missing. EEG based grip strength classification is done using SVM classifier in [5]. Authors claimed higher classification accuracy for strong grip signals due to the higher change in EEG responses in comparison 
to other types of grips. Similar study is done in [15] where authors used time domain features for classification of power of hand grasps. Lili Li et al [13] have performed object oriented motor imagery(OI) analysis using EEG data in four different cases of object, non-object(NI), simple imagery(SI) and visual observation(VO), authors claimed that there is significant suppression in mu rhythm for OI and NI. Sanjay et al [11] have performed an ERP based analysis to examine the EEG based responses to hand actions on objects. Four types of cases have been considered for action analysis-Object with Congruent Grip, Object with In-Congruent Grip and Non object with Congruent and In-Congruent Grip. Authors study shows that even the grip responses were unrelated to the task, ERP responses were sensitive to the type of grip congruency over posterior and motor areas of brain. This work was continued in [12] where Sanjay et al presented a study on mu rhythm de-synchronization over motor areas for different object gripping conditions.

In this paper we are working on the dataset presented by Sanjay et al in [11] [12], in which further analysis of strongly effected electrodes on motor area has been done to analyze the effects of action related information on brain signals by examining the participants decisions to objects with different gripping options. The aim of further analysis is to extract certain effective features and electrodes which can be used for the development of more effective action oriented BCI based devices. We analyzed EEG responses to objects shown with congruent grips, in-congruent grips and without grips. EEG signals are decomposed into frequency bands using DWT and the alpha and beta rhythms are analyzed over both left and right hemisphere electrodes. Further central parietal electrode sites of interest are used for power calculation in all the three cases. It is hoped that the presented study will provide awareness into the role of object affordance during action perception. Further sections of paper are organized as: Section 2 discussed background and related work done with motor related datasets. Section 3 explains the proposed work starting from experimental paradigm, data acquisition to computational analysis of the data. Then Section 4 discusses the results obtained using computation procedure followed by Section 5 which concludes the paper.

\section{Experimental Paradigms and Computational Analysis}

\subsection{Dataset Description}

Participants and EEG Recordings:The presented analysis has been done on EEG dataset by Sanjay et al[11,12] which was recorded continuously with $\mathrm{Ag} / \mathrm{AgCl}$ electrodes placed on 128 scalp locations using nylon electrode cap. The electrodes were placed according to 10-5 electrode system and eight extra electrodes were also used as references, ground, Left and Right Mastoid and for monitoring vertical and horizontal eye movement. The signals were amplified and sampled at a rate of $1024 \mathrm{~Hz}$ using BioSemi Active-Two amplifiers. Dataset was collected by group of 14 people (3male,11female). 
Stimuli and Capturing procedure:For analyzing the effect of action of objects 2D images of 30 objects and non-objects have been used as stimuli. Images of non-objects are generated using Photoshop by combining the part of two objects. Then action based sensory inputs were measured by showing images of object with congruent grip, incongruent grip and no grip. Similarly, participants were shown images for non-objects with congruent, incongruent and no grip as shown in Fig.1. The participants received a total of 180 stimuli of which 30 stimuli pertaining to objects and 30 were for non-object categories.
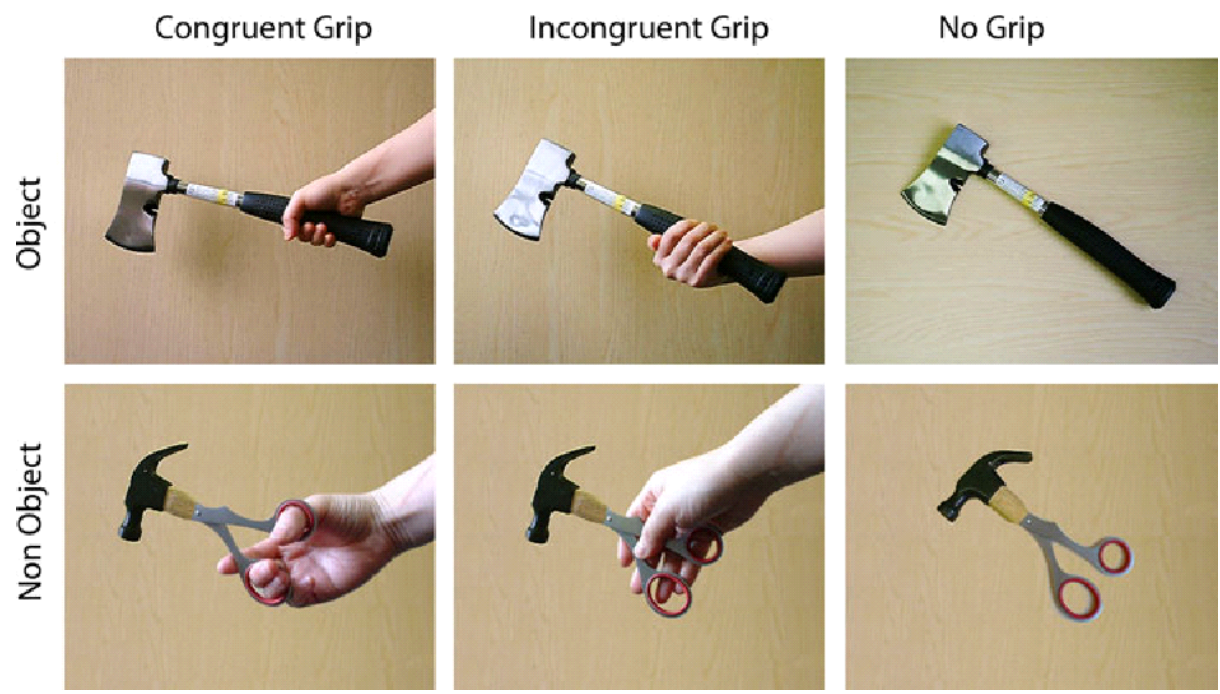

Fig. 1: Images of objects and non-objects stimuli used in experiment

Before each task, the participants received twelve practice trials each of which began with the presentation of a fixation point(x) for $1000 \mathrm{~ms}$ followed by a target stimulus for $1000 \mathrm{~ms}$. Pictorial representation of trials structure is shown in Fig. 2. Participants were asked to make a response within $4000 \mathrm{~ms}$ after stimulus onset. In our work for the perceptual analysis of actions on objects, we have selected the dataset corresponding to 90 stimuli with objects having congruent and in-congruent grip and no grips only. The task was to decide quickly whether the object was a real one or a non-object.

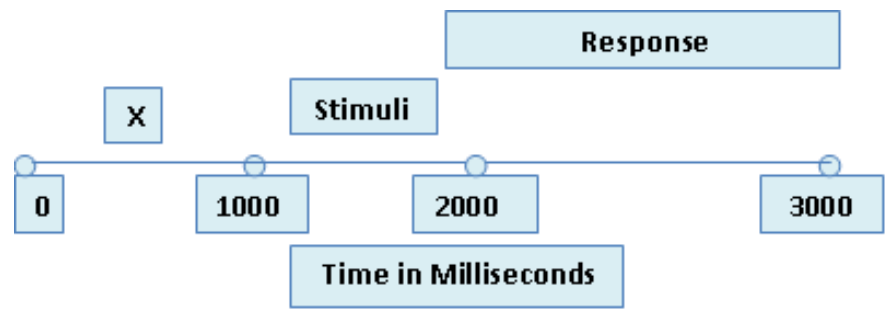

Fig. 2: Trial Structure of each stimulus 


\subsection{Computational Analysis}

The EEG data of 7 participants corresponding to 90 stimuli with objects in three gripping conditions were selected for the analysis. For extracting the distinguishable feature, EEG data was first pre-processed, then alpha and beta band of signals were extracted using DWT based band extraction method. Then baseline normalized power of each band was extracted at different channels of motor area for each gripping conditions. These computational steps are shown in Fig 3 and are discussed below in detail.

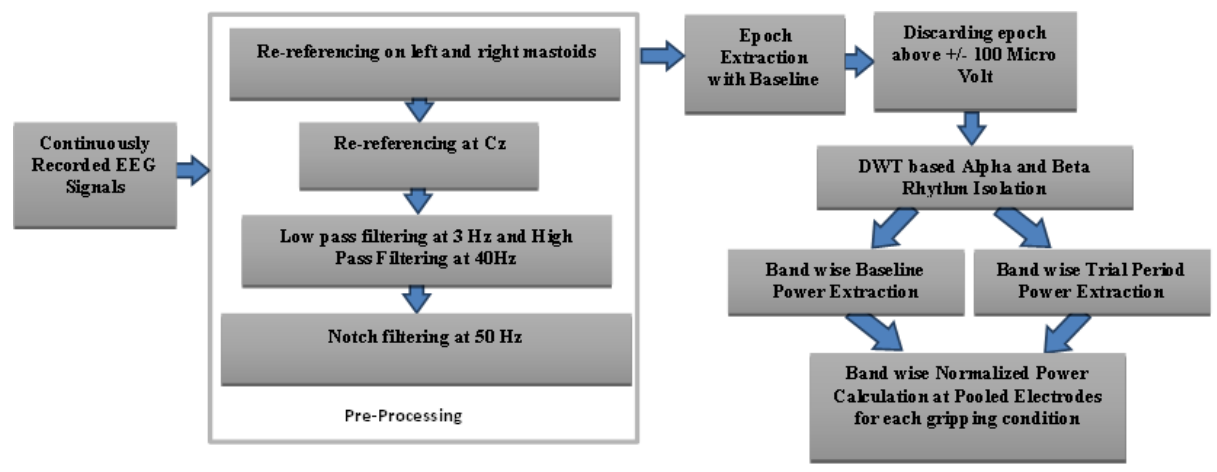

Fig. 3: Procedure for Computational Analysis

Raw EEG Signal: EEG data was recorded continuously using 128 electrodes positions. Original data contains 360 events corresponding to 30 events each for object and non object stimuli and response in three different gripping conditions. Data was sampled at $1024 \mathrm{~Hz}$ and is stored in Biosemi Data (bdf) format.

Pre-Processing: EEG signals are of very poor spatial resolution, have low voltage variations and noise thus requires a lot of pre-processing efforts. Preprocessing procedure of our work is presented in Fig.Inital pre-processing of data was done using EEGLAB software. First data was offline referenced at left (M1) and right mastoid(M2) electrodes to achieve full CMRR, then re-referencing at $\mathrm{Cz}$ position was done to remove CM signal from the data. EEG signals were then band pass filtered from 3 to $40 \mathrm{~Hz}$ and notch filtered at $50 \mathrm{~Hz}$ to remove low frequency and power line noise.

Data Selection: As discussed in section 2, that movement related EEG signals are more dominant in motor cortex region of the brain.EEG data corresponding to 24 electrodes as shown in Fig 4 from each left and right hemisphere of motor area are selected for studying the perceptual changes of effects of different types of gripping.

Epoch Extraction: Continuous selected EEG data is then segmented into epochs of duration 2 seconds (1000 ms before stimuli onset and $1000 \mathrm{~ms}$ after stimuli onset). Epochs were extracted related to 3 different types of events corresponding to Object Congruent, Object In-Congruent and Object No Grip. Further to remove noisy epochs, epochs having values above 100 Micro Volt and below -100 Micro Volt were discarded. $1000 \mathrm{~ms}$ pre-stimuli period is taken as 


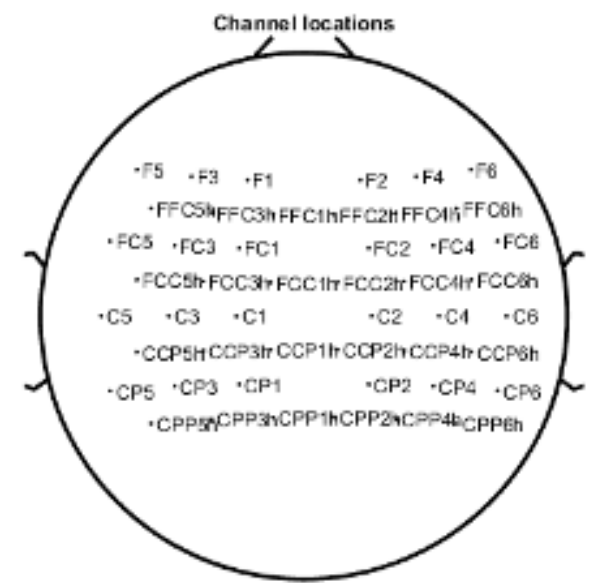

Fig. 4: Distribution of electrodes used for analyzing the motor area

baseline period and $1000 \mathrm{~ms}$ post stimuli period is taken as trial period for each gripping case.

Discrete Wavelet Transform based Alpha, Beta Band Extraction: DWT performs decomposition of time series signals using high pass and low pass filtering with down sampling rate of $2[24,26]$. The high pass filter [HP] is the discrete mother wavelet and the low pass filter $[\mathrm{LP})]$ is its mirror version. The mother wavelet [27] and corresponding scaling function are shown in Figure At each level DWT outputs approximate and detailed coefficients. The approximation coefficients are then further decomposed as shown in Fig. 5 to extract localized information from the sub-band of detail coefficients.

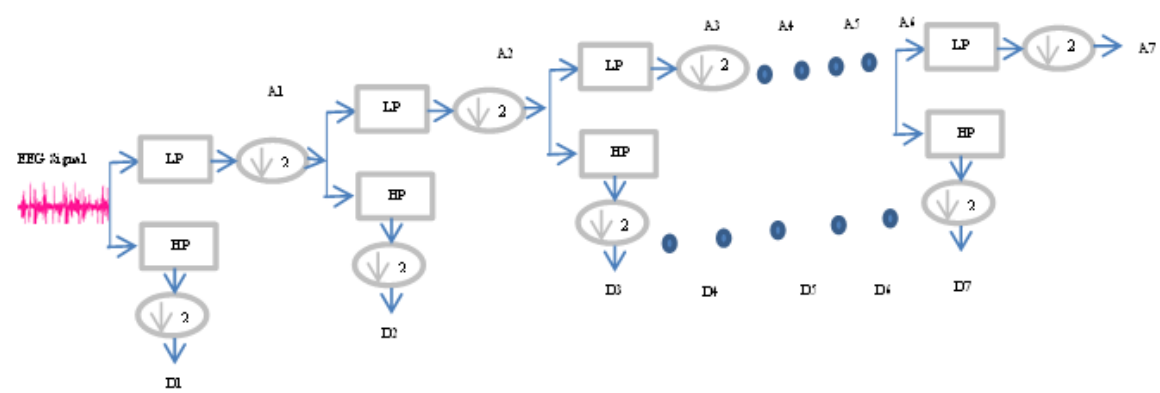

Fig. 5: DWT based signal decomposition into detailed and approximate coefficients

In DWT decomposition levels depends on the dominant frequency component of the signal. In this work EEG data used was sampled at $1024 \mathrm{~Hz}$, thus we have performed 7 level DWT decomposition with Daubechies-eight (db8) mother wavelet. Among extracted sub-bands as given in Table 1 Alpha and Beta bands were used for study of hand grip actions.

Baseline Normalized Power Calculation (BNP):For statistical analysis of different gripping effects on objects, baseline normalized power is calculated using eq. 1 at each channel location and for each gripping condition [18, 25]. 
Table 1: DWT Coefficients and Frequency Bands

\begin{tabular}{|l|l|l|}
\hline \hline DWT Coefficients & Frequency Range & Frequency Bands \\
\hline D1 & $256-512 \mathrm{~Hz}$ & - \\
\hline D2 & $128-256 \mathrm{~Hz}$ & - \\
\hline D3 & $64-128 \mathrm{~Hz}$ & - \\
\hline D4 & $32-64 \mathrm{~Hz}$ & Gamma \\
\hline D5 & $16-32 \mathrm{~Hz}$ & Beta \\
\hline D6 & $8-16 \mathrm{~Hz}$ & Alpha \\
\hline D7 & $4-8 \mathrm{~Hz}$ & Theta \\
\hline A7 & $0-4 \mathrm{~Hz}$ & Delta \\
\hline
\end{tabular}

This normalized power calculates the percentage change in power with respect to baseline, the purpose of normalizing the power with respect to baseline is to remove any activity in the signal that was constant over time (i.e baseline)

$\mathrm{BNP}=100 *$ (Baseline Power-Trial Period Power)/Baseline Power .......(Eq1)

\section{Results and Discussion}

Step wise results of various steps mentioned in Fig 3 are discussed below: After re-referencing, EEG data was filtered to reduce noise. This step is necessary so that meaningful epoch should not be discarded at the time of epoch discarding. After Band pass filtering, EEG data corresponding to 24 electrodes in Fig. 4 was selected for epoch extraction. DWT based band extraction method was
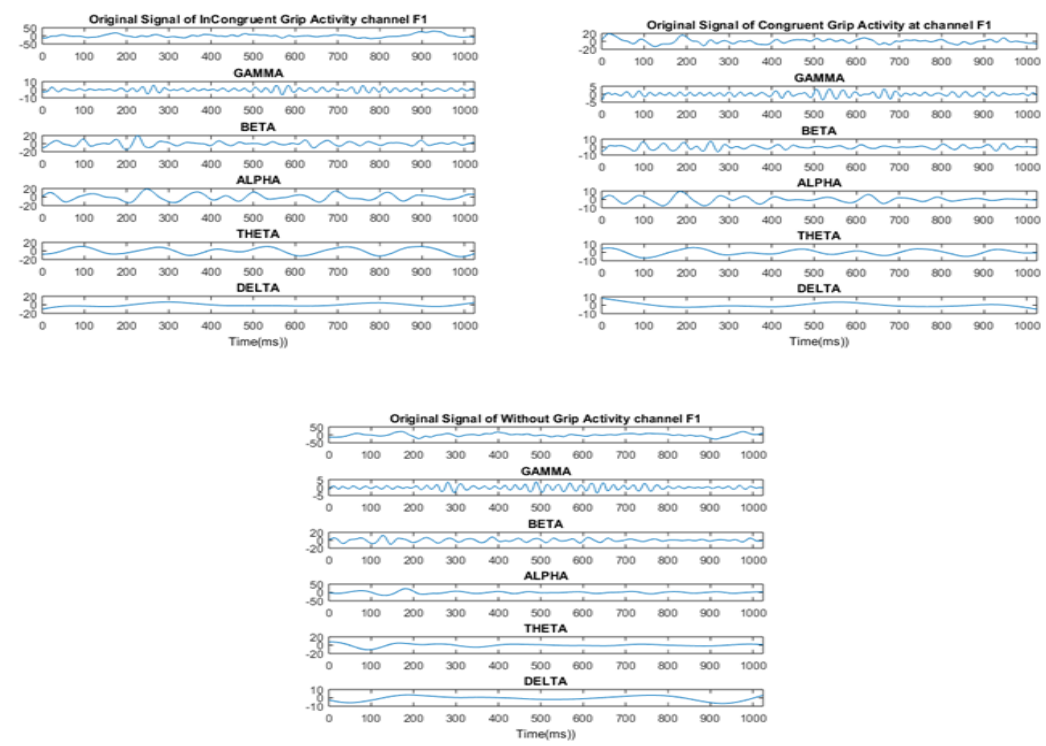

Fig. 6: DWT based Extracted bands (a)Congruent (b)In-Congruent and (c)No Grip Activity 
then applied on epoch data to extract different frequency bands. Fig. 6(a)(b)(c) shows the sample of extracted bands extracted for Congruent, Incongruent and No Grip activity at channel F1.Similar process was done for all the channels, and as any movement related activity over motor area is usually visible in Alpha $(8-12 \mathrm{~Hz})$ and Beta $(12-30 \mathrm{~Hz})$ frequency bands, these bands were chosen for further analysis.

For analyzing the effect in Alpha and Beta bands over three gripping cases, baseline normalized power (BNP) was calculated. The effects were visible on all the mentioned 24 electrodes over motor area, but after analysis it was found there is a significant difference in electrodes over central parietal area as shown in Fig. 7. Since we have considered the data with right hand reaches only, we have taken left side electrodes for further analysis. To find the suitable feature for differentiating three gripping cases, BNP using eq (1) was calculated over following pooled electrodes on Left Hemisphere-

CP1 CP3 CP5 CCP1H CCP3H CCP5H CPP1H CPP3H CPP5H C1 C3 C5

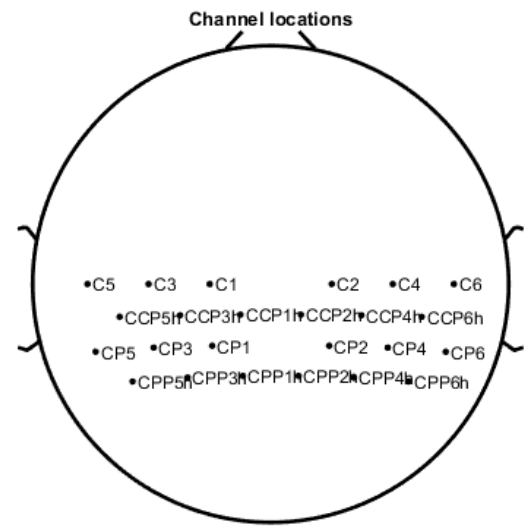

Fig. 7: Distribution of electrodes selected for pooling

Fig. 8 and 9 represents the pooled baseline normalized power (BNP) of all trials of Object with Congruent and In-Congruent grip activity in Alpha and Beta Band respectively. It can be seen from Fig. 8 that BNP for Incongruent Grip is greater than Congruent grip in Alpha Band whereas in beta band (Fig. 9) BNP for Congruent Grip is greater than In-Congruent Grip.

Similar analysis was done for Object with Congruent and Without grip activity. Results presented in Fig. 10 shows there BNP is high in Beta Band for Congruent grip activity than without grip activity, whereas little difference can be seen in Alpha Band (Fig.11).

The average Alpha and Beta band baseline normalized power of pooled electrodes for subject 1 across all trails in Congruent, Incongruent and without grip activity is given in Table 2. Mentioned analysis on the basis of these results is summarized Table 3. 


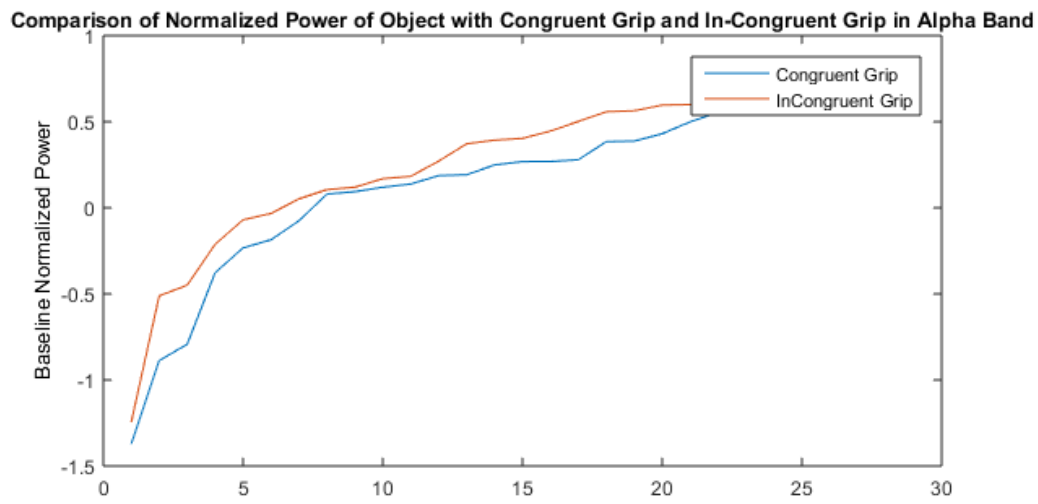

Fig. 8: Comparison of BNP of Congruent and Incongruent Grip Activity in Alpha Band

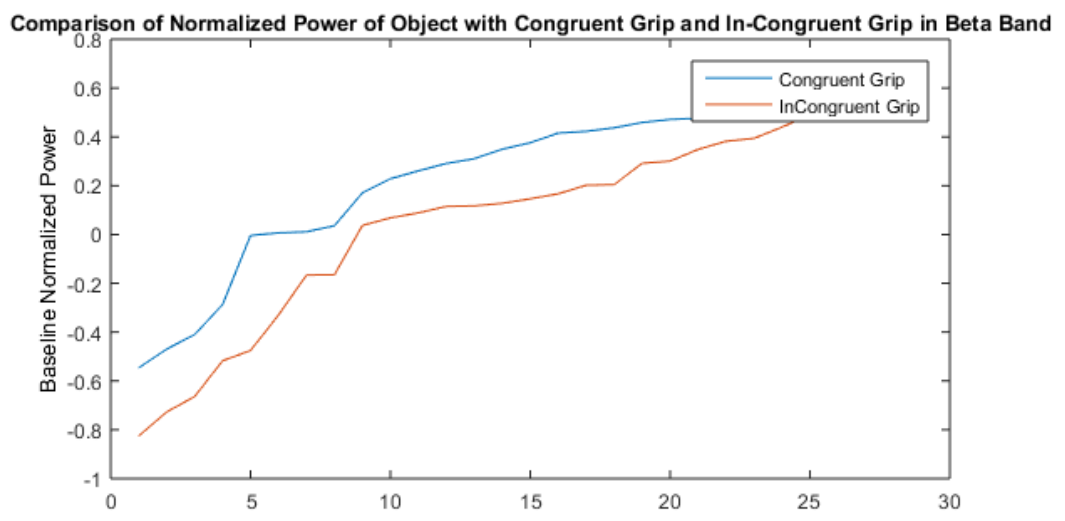

Fig. 9: Comparison of BNP of Congruent and Incongruent Grip Activity in Beta Band

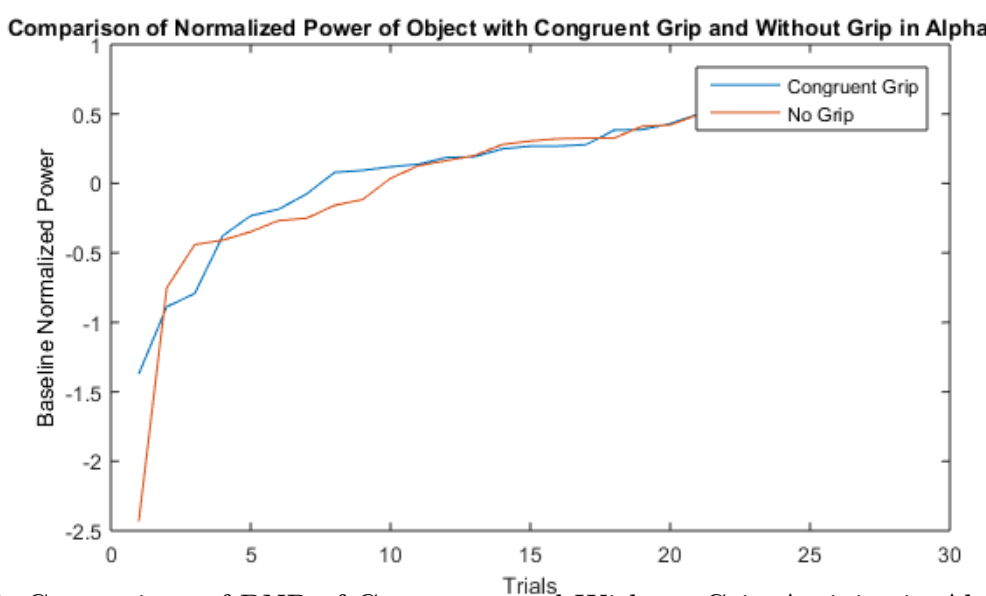

Fig. 10: Comparison of BNP of Congruent and Without Grip Activity in Alpha Band 


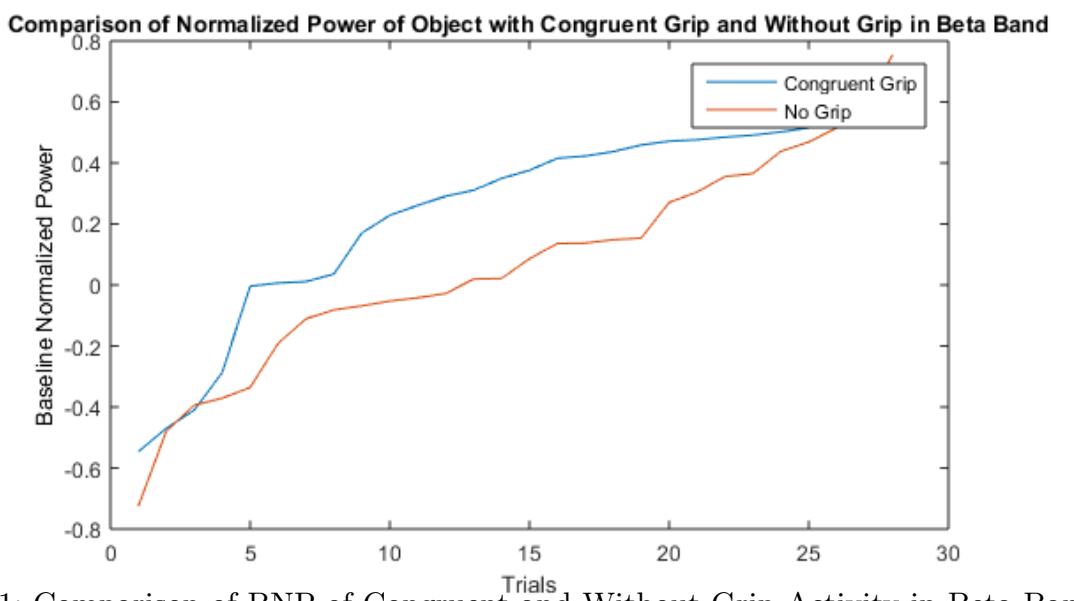

Fig. 11: Comparison of BNP of Congruent and Without Grip Activity in Beta Band

Table 2: Average BNP over pooled electrodes in three different gripping cases

\begin{tabular}{|l|l|l|}
\hline \hline $\begin{array}{l}\text { Frequency Band for } \\
\text { BNP }\end{array}$ & Congruent Grip & In-Congruent Grip \\
No Grip & \multicolumn{2}{|l|}{} \\
\hline Alpha Power & 23.4520 & 43.3371 \\
28.7069 & 28.7648 & 12.3537 \\
\hline Beta Power & & \\
15.7364 & & \multicolumn{2}{|l}{} \\
\hline \hline
\end{tabular}

Table 3: Analysis of effect of different gripping cases

\begin{tabular}{|l|l|l|}
\hline \hline $\begin{array}{l}\text { Power at Pooled } \\
\text { Electrodes }\end{array}$ & $\begin{array}{l}\text { Congruent Grip(C) } \\
\text { and In-Congruent } \\
\text { Grip(IC) }\end{array}$ & $\begin{array}{l}\text { Congruent Grip(CG) } \\
\text { and No Grip(NG) }\end{array}$ \\
\hline $\begin{array}{l}\text { Alpha Power (Base- } \\
\text { line Normalized) }\end{array}$ & $\mathrm{C}<\mathrm{IC}$ & $\begin{array}{l}\text { A little difference } \\
\text { which cannot be } \\
\text { differentiated }\end{array}$ \\
\hline $\begin{array}{l}\text { Beta Power (Baseline } \\
\text { Normalized) }\end{array}$ & $\mathrm{C}>\mathrm{IC}$ & $\mathrm{C}>\mathrm{NG}$ \\
\hline \hline
\end{tabular}

\section{Conclusion}

In this work EEG based analysis has been done to analyse the effect of object affordance on object perception and recognition. Baseline normalized power in Alpha and Beta bands were examined in three different object gripping cases i.e Congruent, In-Congruent gripping No gripping over motor areas. Further to find the distinguishable feature 12 electrodes over central parietal areas were selected. 
The result shows that the effect of object with correct grip and without grip is clearly visible in Alpha band, whereas difference between object with correct grip and incorrect grip can be seen in both Alpha and Beta band. The presented study and features can be used for the development of automatically classifying EEG signals, which can be used to develop various brain machine interfaces for neurorehabilitation purpose. It is hoped that the presented study will provide awareness into the role of object affordance during action perception.

\section{References}

1. Gibson, James J., The Theory of Affordances The Ecological Approach to Visual Perception. Boston:Houghton Mifflin, 1979. Print.

2. Norman, D. A. (1988). The psychology of everyday things. New York: Basic Books.

3. Isaiah Inns, Elizabeth DeSilva, A proposed EEG study: the role of object affordance during action observation, Indiana University Journal of Undergraduate Research(IUJUR) Volume III, 2017, pp:44-47

4. Hassanien, Aboul Ella Azar, Ahmad. Brain computer interface: trends and applications. Intelligent Systems Reference Library, vol. 74. Springer (2015).

5. Kim D-E, Yu J-H, Sim K-B. EEG Feature Classification Based on Grip Strength for BCI Applications. International Journal of Fuzzy Logic and Intelligent Systems. $2015 ; 15(4): 277-282$

6. Rupal Chaudhari, Hiren J. Galiyawala, A Review on Motor Imagery Signal Classification for BCI, Signal Processing: An International Journal (SPIJ), Volume (11) : Issue (2) : 2017.

7. Hafeez Ullah Amin, Wajid Mumtaz, Ahmad Rauf Subhani, Mohamad Naufal Mohamad Saad and Aamir Saeed Malik, Classification of EEG Signals Based on Pattern Recognition Approach, Front. Comput. Neurosci., 21 November 2017.

8. S. H. Choi, M. Lee, Y. Wang and B. Hong, "Estimation of Optimal Location of EEG Reference Electrode for Motor Imagery Based BCI Using fMRI," 2006 International Conference of the IEEE Engineering in Medicine and Biology Society, New York, NY, 2006, pp. 1193-1196.

9. Stefanie Schuch, Andrew P. Bayliss, Christoph Klein, Steven P. Tipper, Attention modulates motor system activation during action observation: evidence for inhibitory rebound, Exp Brain Res (2010) pp:235249.

10. Oberman LM, Pineda JA, Ramachandran VS. The human mirror neuron system: A link between action observation and social skills. Social cognitive and affective neuroscience. 2007;2(1):62-66. doi:10.1093/scan/ns1022.

11. Sanjay Kumar, Eun Young Yoon Glyn W. Humphreys, Perceptual and motorbased responses to hand actions on objects: evidence from ERPs, Experimental Brain Research, 2012 Jul;220(2):153-64

12. Kumar, Sanjay Jane Riddoch, Margaret Humphreys, Glyn. (2013). Mu rhythm desynchronization reveals motoric influences of hand action on object recognition. Frontiers in human neuroscience. 7. 66. 10.3389/fnhum.2013.00066.

13. Li L, Wang J, Xu G, Li M, Xie J (2015) The Study of Object-Oriented Motor Imagery Based on EEG Suppression. PLoS ONE 10(12): e0144256. doi:10.1371/journal.pone.0144256.

14. S. R. Sreeja, Joytirmoy Rabha, Debasis Samanta, Pabitra Mitra, and Monalisa Sarma , Classification of Motor Imagery Based EEG Signals Using Sparsity Approach, IHCI 2017,pp. 4759 
15. Roy, Rinku Sikdar, Debdeep Mahadevappa, Manjunatha Cheruvu, Kumar. (2017). EEG Based Motor Imagery Study of Time Domain Features for Classification of Power and Precision Hand Grasps, 8th International IEEE EMBS Conference on Neural Engineering Shanghai, China, May 25 - 28 , 2017

16. A.Sivakami and S.Shenbaga Devi, Analysis of EEG for motor imagery based classification of hand activities, International Journal of Biomedical Engineering and Science (IJBES), Vol. 2, No. 3, July 2015.

17. Gerrit Lange, Cheng Yee Low, Khairunnisa Johar, Fazah Akthar Hanapiah, Fadhlan Kamaruzaman, Classification of Electroencephalogram Data from Hand Grasp and Release Movements for BCI Controlled Prosthesis, Procedia Technology, Volume 26,2016, Pages 374-381.

18. Mohammad H. Alomari, Aya Samaha, Khaled AlKamha, Automated Classification of L/R Hand Movement EEG Signals using Advanced Feature Extraction and Machine Learning, International Journal of Advanced Computer Science and Applications (IJACSA) 07/2013; 4(6):207-212

19. A.Sivakami and S.Shenbaga Devi, Analysis of EE for Motor Imagery based Classification of hand activities, International Journal of Biomedical Engineering and Science (IJBES), Vol. 2, No. 3, July 2015.

20. S. S. Gupta and S. Agarwal, "Classification and analysis of EEG signals for imagined motor movements," 2015 IEEE Workshop on Computational Intelligence: Theories, Applications and Future Directions (WCI), Kanpur, 2015, pp. 1-7.

21. Jun Matsumoto, Toshiyuki Fujiwara, Osamu Takahashi, Meigen Liu, Akio Kimura and Junichi Ushiba, Modulation of mu rhythm desynchronization during motor imagery by transcranial direct current stimulation, Journal of NeuroEngineering and Rehabilitation, 2010.

22. Patricia Batres-Mendoza, Mario A. Ibarra-Manzano, Erick I. Guerra-Hernandez, Dora L. Almanza-Ojeda, Carlos R. Montoro-Sanjose, Rene J.Romero-Troncoso, and Horacio Rostro-Gonzalez, Improving EEG-Based Motor Imagery Classification for Real-Time Applications Using the QSA Method, Computational Intelligence and Neuroscience, Volume 2017.

23. D.Hari Krishnaa, I.A.Pashaa, T.Satya Savithrib, Classification of EEG Motor imagery multi class signals based on Cross Correlation, International Conference on Computational Modelling and Security, CMS 2016.

24. E. L. A. Vivas, A. Garca-Gonzlez, I. Figueroa and R. Q. Fuentes, "Discrete Wavelet transform and ANFIS classifier for Brain-Machine Interface based on EEG," 2013 6th International Conference on Human System Interactions (HSI), Sopot, 2013, pp. $137-144$.

25. Shedeed, H.A., Issa, M.F.: Brain-EEG signal classification based on data normalization for controlling a robotic arm. Int. J. Tomogr. Simul. 29, 7285 (2016).

26. Subasi, A. (2007). EEG signal classification using wavelet feature extraction and a mixture of expert model. Expert Syst. Appl. 32, 10841093. doi: 10.1016/j.eswa.2006.02.005

27. (2018) Article on Wavelet db8, [Online] Available: http://wavelets.pybytes.com/wavelet/db8/

28. Mike X Cohen, Chapter 18-Analyzing neural time series data: Theory and practice (2014), MIT Press. 